\title{
Hydrogenization of underground storage of natural gas
}

\author{
Impact of hydrogen on the hydrodynamic and bio-chemical behavior
}

\author{
B. Hagemann ${ }^{1,2} \cdot$ M. Rasoulzadeh ${ }^{2}$ M. Panfilov ${ }^{2}$ L. Ganzer ${ }^{1} \cdot$ V. Reitenbach ${ }^{1}$
}

Received: 25 December 2014 / Accepted: 14 July 2015 / Published online: 22 August 2015

(C) The Author(s) 2015. This article is published with open access at Springerlink.com

\begin{abstract}
The intermittent production of the renewable energy imposes the necessity to temporarily store it. Large amounts of exceeding electricity can be stored in geological strata in the form of hydrogen. The conversion of hydrogen to electricity and vice versa can be performed in electrolyzers and fuel elements by chemical methods. The nowadays technical solution accepted by the European industry consists of injecting small concentrations of hydrogen in the existing storages of natural gas. The progressive development of this technology will finally lead to the creation of underground storages of pure hydrogen. Due to the low viscosity and low density of hydrogen, it is expected that the problem of an unstable displacement, including viscous fingering and gravity overriding, will be more pronounced. Additionally, the injection of hydrogen in geological strata could encounter chemical reactivity induced by various species of microorganisms that consume hydrogen for their metabolism. One of the products of such reactions is methane, produced from Sabatier reaction between $\mathrm{H}_{2}$ and $\mathrm{CO}_{2}$. Other hydrogenotrophic reactions could be caused by acetogenic archaea, sulfate-reducing bacteria and iron-reducing bacteria. In the present paper, a mathematical model is presented which is capable to reflect
\end{abstract}

B. Hagemann

birger.hagemann@tu-clausthal.de

1 Clausthal University of Technology, Clausthal-Zellerfeld, Germany

2 Laboratoire d'Energétique et de Mécanique Théorique et Appliquée, Université de Lorraine/CNRS, Vandœuvre-lèsNancy, France the coupled hydrodynamic and bio-chemical processes in UHS. The model has been numerically implemented by using the open source code DuMuX developed by the University of Stuttgart. The obtained bio-chemical version of DuMuX was used to model the evolution of a hypothetical underground storage of hydrogen. We have revealed that the behavior of an underground hydrogen storage is different than that of a natural gas storage. Both, the hydrodynamic and the bio-chemical effects, contribute to the different characteristics.

Keywords Underground hydrogen storage - Microbial population dynamics - Numerical modeling - Displacement instability $\cdot$ Methanogenesis

\section{Introduction}

Underground hydrogen storage (UHS) is a promising answer for the balance of electrical energy related to the fluctuating supply from renewable energy sources. The renewable energy supply which is generated primarily by wind and solar power plants is strongly dependent on weather conditions. Additionally, the demand of electricity varies on a daily and seasonal time scale. The balance implies the temporal storage of electricity wherefore several storage options are currently under discussion [21]. Underground hydrogen storages where the energy is stored as chemical energy have a high energy density and consequently they provide the possibility to store electrical energy in the long term or seasonal period [9]. The technology comprises electrolyzers which use excessive electrical energy to split water into oxygen and hydrogen [21]. Different concepts for the subsequent storage and usage of hydrogen are available $[9,17,27]$ : 
- "POWER-to-GAS": The produced hydrogen will be fed into the existing natural gas grid. Investigations have shown that concentrations in the single-digit percentage area are supposable [13]. This means that also the existing underground gas storages will be charged with natural gas containing low percentages of hydrogen.

- "POWER-to-GAS-to-POWER": The hydrogen is stored purely in subsurface formations such as depleted gas or oil reservoirs, aquifers, and solution mined caverns [32]. At times of energy demand, the hydrogen is withdrawn and can be used as energy fuel for stationary fuel cells or engine-generators connected to the electrical grid or for fuel cell vehicles.

In previous publications, it was stated that UHS is practically identical to the storage of natural gas [5, 6]. However, in the present paper, we show that there are large differences which arise due to the particular hydrodynamic and bio-chemical characteristics. A coupled hydrodynamic and bio-chemical model was developed, numerically implemented and used for the investigation of a case study.

\section{Hydrodynamic behavior in UHS}

The operation of cyclic hydrogen storage is similar to the intermediate storage of natural gas which is required for the daily and seasonal balance. However, the hydrodynamic characteristics of hydrogen are different. Especially in porous rock formations, its behavior is insufficiently studied. Hydrogen has a very low density and viscosity which involves a high tendency for an unstable displacement including gravity overriding and viscous fingering during the injection period. The mobility ratio for the displacement of water by hydrogen is in the order of 100 and consequently highly unfavorable. This problem was considered by [30]. He concluded that in anticlinal aquifer storages, viscous fingers could spread laterally beyond the spill point and unrecoverable losses of hydrogen could occur. A control parameter for this behavior is the injection rate. During slow hydrogen injection, the gravitational forces dominate and stabilize the displacement while a fast injection leads to the domination of viscous forces and consequently an unstable displacement or viscous fingering. Hence, a limitation on the injection rate could reduce the hydrogen losses. Additionally, hydrogen has a high diffusivity whose impact was analyzed in the paper of [6]. They stated that recognizable amounts of hydrogen can get lost due to dissolution into the connate water and diffusion through the cap rock and into the surrounding aquifer.

\section{Bio-chemical reactions in UHS}

Hydrogen has a high reactivity and is able to undergo a large number of chemical compounds. Possible reactants in underground storages are sulfides, sulfates, carbonates, and oxides which are present in the reservoir rocks [16] or hydrocarbons, carbon dioxide, and dissolved components which are present in the fluid phases. However, the usual temperature and $\mathrm{pH}$ conditions in the reservoir are not adequate for these reactions and therefore a catalysis by microorganisms is required [35]. Hydrogen is a universal electron donor for the metabolism of different anaerobic microbial species (including archaea and bacteria) which are present in subsurface formations. Evidence for this behavior is given by observations in some of the former town gas storages. The storage near Lobodice, Czech Republic showed a drastic increase in the $\mathrm{CH}_{4}$ concentration and a decrease in the $\mathrm{CO}, \mathrm{CO}_{2}$, and $\mathrm{H}_{2}$ concentrations during a storage cycle of 7 months [33]. [33] analyzed the existence of methanogenic archaea in the formation water and concluded that methane was produced in situ by biochemical reactions. The activity of sulfate-reducing bacteria was also observed in town gas and natural gas storages. Indications are often the production of $\mathrm{H}_{2} \mathrm{~S}$ and the resulting corrosion problems [22]. Other sources [8] and [23] give hints that also acetogenic archaea and iron-reducing bacteria could be stimulated and contribute in the metabolism of hydrogen.

According to the available literature, it is expected that four hydrogenotrophic microbial processes could be important for UHS:

- Methanogenesis:

$$
\mathrm{CO}_{2}+4 \mathrm{H}_{2} \longrightarrow \mathrm{CH}_{4}+2 \mathrm{H}_{2} \mathrm{O}
$$

where $\mathrm{H}_{2}$ and $\mathrm{CO}_{2}$ are present in the liquid and gas phase.

- Acetogenesis:

$$
2 \mathrm{CO}_{2}+4 \mathrm{H}_{2} \longrightarrow \mathrm{CH}_{3} \mathrm{COOH}+2 \mathrm{H}_{2} \mathrm{O}
$$

- Sulfate-reduction:

$$
\mathrm{SO}_{4}^{2-}+5 \mathrm{H}_{2} \longrightarrow \mathrm{H}_{2} \mathrm{~S}+4 \mathrm{H}_{2} \mathrm{O}
$$

where $\mathrm{SO}_{4}^{2-}$ is dissolved in water.

- Iron(III)-reduction:

$$
3 \mathrm{Fe}_{2}^{\mathrm{III}} \mathrm{O}_{3}+\mathrm{H}_{2} \longrightarrow 2 \mathrm{Fe}_{3}^{\mathrm{II}} \mathrm{O}_{4}+\mathrm{H}_{2} \mathrm{O}
$$

where iron-oxides are present in the reservoir rocks.

Other variations of the reaction equations are possible; however, the stated equations represent the microbial processes in an adequate way. The coexistence of several species can thereby result in a concurrence between the different hydrogenotrophic processes which was investigated by [8] 
and [23]. They have shown that a simultaneous survival and also an out-competition is possible. The microorganisms have revealed to have different minimum threshold concentrations for the consumption of hydrogen. Iron-reducing bacteria have the lowest threshold concentration and consequently the best potential to out-compete other species. However, when hydrogen is available in excessive amount, several species can reproduce simultaneously depending on the presence of the corresponding electron acceptor.

\section{Two-phase bio-reactive transport model}

The modeling of UHS includes several physical, chemical, and biological processes. In the literature, two bio-reactive transport models are available which were developed for the application to UHS: [14] developed a model at pore scale while [29] and [34] developed a model at macroscopic scale. Additionally, there is a large number of models for bio-reactive transport in groundwater which are constrained to single-phase flow. An overview of modeling approaches can be found in the papers of [18] and [28]. In the recent paper, a new model is developed at macroscopic scale which couples the flow and transport of two mobile phases to the bio-chemical processes of multiple microbial species.

\subsection{Physico-chemical processes}

The reservoir is described as porous medium which is saturated by up to two phases, gas and water. At macroscopic scale, the porous medium and all present fluids can be treated as continuous equivalent media. Hence, the overall conservation of mass for each chemical component can be written in the following form whereby advective and dispersive/diffusive transport is considered ([7]):

$$
\begin{aligned}
& \phi \frac{\partial\left(\rho_{g} c_{g}^{k} S_{g}+\rho_{w} c_{w}^{k} S_{w}\right)}{\partial t} \\
& +\nabla \cdot\left(\rho_{w} c_{w}^{k} v_{w}+J_{w}^{k}+\rho_{g} c_{g}^{k} v_{g}+J_{g}^{k}\right)=q^{k}
\end{aligned}
$$

where $\phi$ is the porosity, $\rho$ is the molar density in $\left(\mathrm{mol} / \mathrm{m}^{3}\right)$, $c$ is the mole fraction, $S$ is the saturation, $v$ is the advective flux in $(\mathrm{m} / \mathrm{s}), J$ is the dispersive/diffusive flux in $\left(\mathrm{mol} / \mathrm{m}^{2} / \mathrm{s}\right)$, and $q$ is a source or sink term. The subscripts $g$ and $w$ denote the gas and water phase respectively and the superscript $k$ refers to the chemical component. Equivalent the conservation of mass can be written for immobile solid components by only considering the rate of reaction:

$$
(1-\phi) \frac{\partial\left(\rho_{s} c_{s}^{k}\right)}{\partial t}=q^{k}
$$

where the subscript $s$ denotes the solid phase.
The volumetric velocity is formulated by Darcy's law:

$v_{i}=-\frac{K k_{r i}}{\mu_{i}} \cdot\left(\nabla P_{i}-\hat{\rho}_{i} g\right), \quad i=g, w$

where $K$ is the absolute permeability in $\left(\mathrm{m}^{2}\right), k_{r}$ is the relative permeability, $\mu$ is the dynamic viscosity in $(P a \cdot s), P$ is the phase pressure in $(\mathrm{Pa}), \hat{\rho}$ is the phase density in $\left(\mathrm{kg} / \mathrm{m}^{3}\right)$, and $g$ is the gravity acceleration in $\left(\mathrm{m} / \mathrm{s}^{2}\right)$.

For the dispersion/diffusion term, it needs to be distinguished between the phases. In the water phase, it is formulated by Fick's law considering molecular diffusion and mechanical dispersion:

$J_{w}^{k}=-\rho_{w}\left(D_{\text {diff }, w}^{k}+D_{\text {disp }, w}^{k}\right) \nabla c_{w}^{k}$

where $D_{\text {diff, } w}^{k}$ is the effective molecular diffusion coefficient of component $k$ in water in $\left(\mathrm{m}^{2} / \mathrm{s}\right)$ and $D_{\mathrm{disp}, w}^{k}$ is the mechanical dispersion coefficient of component $k$ in water in $\left(\mathrm{m}^{2} / \mathrm{s}\right)$. In contrast, the molecular diffusion in the gas phase is formulated by Stefan-Maxwell equation which was simplified by Blanc's law [31]:

$J_{g}^{k}=-\left(\sum_{j=1 \neq i}^{n} \frac{c_{g}^{j}}{\rho_{g} D_{\mathrm{diff}, g}^{i j}}\right)^{-1} \nabla c_{g}^{k}-\rho_{g} D_{\mathrm{disp}, g}^{k} \nabla c_{g}^{k}$

where $D_{\text {diff, } g}^{i j}$ is the effective binary diffusion coefficient between component $i$ and component $j$ in $\left(\mathrm{m}^{2} / \mathrm{s}\right)$.

The mechanical dispersion coefficient is defined dependent on the alignment to the flow direction (10):

$D_{\mathrm{disp}, i}^{k}=\frac{v_{i} v_{i}^{T}}{\left\|v_{i}\right\|}\left(\alpha_{L}-\alpha_{T}\right)+\left\|v_{i}\right\| \alpha_{T}$

where $\alpha_{L}$ is the longitudinal dispersivity in (m) and $\alpha_{T}$ is the transverse dispersivity in $(\mathrm{m})$.

The hydraulic properties (capillary pressure and relative permeability) of the porous medium are calculated by using the Brooks-Corey correlation [4]:

$P_{c}\left(S_{w}\right)=P_{g}-P_{w}=P_{e} S_{w e}^{-\frac{1}{\lambda}}$

$k_{r w}\left(S_{w}\right)=S_{w e}^{\frac{2+3 \lambda}{\lambda}}$

$k_{r g}\left(S_{w}\right)=\left(1-S_{w e}\right)^{2}\left(1-S_{w e}^{\frac{2+\lambda}{\lambda}}\right)$

where $P_{e}$ is the entry capillary pressure in $(\mathrm{Pa})$ and $S_{w e}$ is the effective water saturation:

$S_{w e}=\frac{S_{w}-S_{w r}}{1-S_{w r}-S_{g r}}$

where $S_{w r}$ is the residual water saturation and $S_{g r}$ is the residual gas saturation.

The mass exchange between the phases is modeled by thermodynamic equilibrium conditions. This assumption is 
usually used in petroleum reservoir and geochemical simulations because the mass exchange between the phases takes place much faster than the chemical reactions and the flux. However, for bio-chemical reactions, the rates could be much faster what implies a limiting factor for this model. The range of validity for this assumption is discussed in detail in the paper of [19]. The thermodynamic equilibrium condition in this model is defined by the equality of fugacities:

$f_{g}^{k}=f_{w}^{k} \quad$ or $\quad c_{g}^{k} \varphi_{g}^{k} P_{g}=c_{w}^{k} \varphi_{w}^{k} P_{w}$

where $f$ is the fugacity in (Pa) and $\varphi$ is the fugacity coefficient. The gas phase is treated as ideal gas what means the the fugacity coefficients are equal to 1 , whereas in the water phase the fugacity coefficients are calculated by Henry's law. By using an adequate correlation for the Henry constants, this method predicts the thermodynamic equilibrium for a gas-water system with a very low error [31]. The hydrodynamic properties of the phases (density, dynamic viscosity) are correlated related to pressure, temperature, and composition.

The system of equations is closed by the sum of saturations and the sum of concentrations:

$$
S_{g}+S_{w}=1 \quad \sum_{k} c_{g}^{k}=1 \quad \sum_{k} c_{w}^{k}=1
$$

\subsection{Bio-chemical processes}

Microbial populations in porous media can live in different structures. In a porous medium, saturated by gas and water, three different structures are feasible $[1,18]$ :

- Aqueous/Plankton: The microorganisms are freely living in the water phase. In this structure, the microorganisms are transported with the flow of water.

- Solid-liquid biofilm: The microorganisms are living in a biofilm attached to the pore walls in the water wet porous medium.

- Gas-liquid (neuston) biofilm: The microorganisms are living in a biofilm in the direct proximity to the gaswater interface (at pore-scale). In this structure, the microorganisms are transported with the movement of the gas-water interface.

In each of these structures, a growth and decay of microorganisms takes place dependent on the availability of substrates and electron acceptors. Microorganisms can attach and detach which means that an exchange between the different structures occurs. Additionally, a movement of microorganisms takes place by random motion (similar to diffusion) and chemotaxis which means that the microorganisms are moving towards the higher substrate concentration.
At macroscopic scale, some simplifications are required for a proper representation. The present model uses an unstructured approach for the microbial populations which means that only one general structure is used for all microorganisms present. Additionally, the advective and chemotactic transport of microorganisms is neglected. Hence, the microbial population dynamics can be formulated by:

$$
\begin{aligned}
\frac{\partial n}{\partial t}= & S_{w} \psi^{\text {growth }}\left(c_{w}^{S}, c_{w}^{A}\right) \cdot n-\psi^{\text {decay }} \cdot n \\
& +\nabla \cdot\left(D_{m} \nabla n\right)
\end{aligned}
$$

where $n$ is the number of microorganisms in $\left(1 / \mathrm{m}^{3}\right), \psi^{\text {growth }}$ is the microbial growth function in $(1 / \mathrm{s})$ which is a function of the substrate concentration $c_{w}^{S}$ and the electron acceptor concentration $c_{w}^{A}$ in the water phase, $\psi^{\text {decay }}$ is the decay function in (1/s) and $D_{m}$ is the microbial diffusion coefficient in $\left(\mathrm{m}^{2} / \mathrm{s}\right)$. Furthermore, it is assumed that the number of microorganisms has no influence on the hydrodynamic parameters (porosity, absolute and relative permeabilities) of the porous medium. Certainly, this assumption has a lack and therefore it is planned to cover these influences in the ongoing research.

Different models for the growth and decay function are available in the literature which are discussed in Section 4.3.

The degradation of substrates is directly linked to the microbial growth. Hence, it is reasonable to use a proportional relation between the rate of growth and the rate of the bio-chemical reaction:

$q^{k}=\phi S_{w} \gamma^{k} \frac{\psi^{\text {growth }}}{Y} n$

where $\gamma$ is the stoichiometric coefficient related to the reaction equations (1) to (4) in (mol) and $Y$ is the yield coefficient which relates the reproduction of microorganisms to the consumption of substrates.

\subsection{Review of models for microbial growth and decay}

It is important to describe the growth and decay of the microorganisms in an adequate way because this is related to the local rates of the bio-chemical reactions. Analogies can be taken from batch culture experiments, where the microorganisms are exposed to an initially added amount of substrate. [25] described the life cycle of a microbial batch culture with up to six phases: (1) the lag, (2) the acceleration, (3) the exponential growth, (4) the deceleration, (5) the stationary, and (6) the decay phase. In Fig. 1, a typical growth function of a batch culture is plotted. It shows the number of microorganisms in a logarithmic scale versus time. However, growth functions can look more simple when one or more of the phases are absent.

Several mathematical models exist in the literature to describe the behavior of the microbial growth function. 


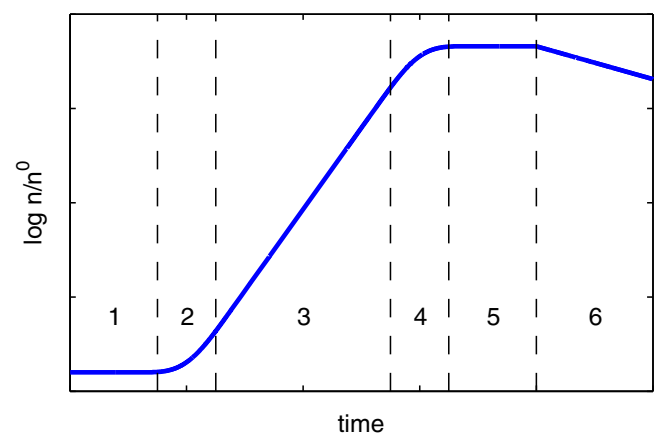

Fig. 1 Life cycle of a microbial batch culture (redrawn after [25])

Classical models consist of a single function which is adjustable by some empirical parameters. More consistent models with the UHS conditions are also taking into account the availability of substrates. Such models are called substrate-limited growth models. Examples are the Monod model, the Moser model, and the recent developed model for UHS [29]:

- Monod model [25]:

$$
\psi^{\text {growth }}=\psi_{\max }^{\text {growth }}\left(\frac{c^{S}}{\alpha+c^{S}}\right)
$$

where $\psi_{\max }^{\text {growth }}$ is the maximum specific growth rate in $[1 / s], c^{S}$ is the concentration of the limiting substrate, and $\alpha$ is the half-velocity constant.

- Moser model [26]:

$$
\psi^{\text {growth }}=\psi_{\max }^{\text {growth }}\left(\frac{\left(c^{S}\right)^{\eta}}{\alpha+\left(c^{S}\right)^{\eta}}\right)
$$

where $\eta$ is an additional exponent compared to the Monod model.

- Panfilov model [29]:

$$
\psi^{\text {growth }}=\frac{1}{t_{e}} \frac{n}{1+\frac{n^{2}}{n_{\max }^{2}}}\left(\frac{c^{S}}{\alpha+c^{S}}\right)
$$

where $t_{e}$ is the characteristic time of eating in (s) and $n_{\max }$ is the maximum population size in $\left(1 / \mathrm{m}^{3}\right)$.

The decay rate can also be modeled in different ways. The simplest method is to use a constant decay rate. Further, it is possible to introduce a decay rate which depends linearly on the number of microorganisms:

- Constant decay rate:

$$
\psi^{\text {decay }}=d
$$

- Increasing decay rate:

$$
\psi^{\text {decay }}=d \cdot n
$$

where $d$ is the decay coefficient.

A batch culture experiment can be simulated by the following pair of ordinary differential equations:

$\frac{\partial n}{\partial t}=\psi^{\text {growth }} \cdot n-\psi^{\text {decay }} \cdot n$

$\frac{\partial c^{S}}{\partial t}=-\frac{\psi^{\text {growth }}}{Y} \cdot n$

In Fig. 2, the qualitative behavior of the introduced models is compared.

It should be mentioned that neither of these models is able to predict the lag and stationary phases. For all of them, the growth starts immediately and the deceleration phase continues directly in the decay phase. Only the model of [29] shows the acceleration phase. The Monod and Moser model start directly with the exponential growth phase. From laboratory experiments, it is reported that the lag phase can be in the range of completely absent up to some weeks [12]. In situ, the conditions are different and the lag phase could be even longer. Consequently, its consideration could be quite important.

However, these models do not take into account the competitive inhibition which could occur when several microbial species compete for the same substrate. An extended
Fig. 2 Comparison of different models for microbial population dynamics

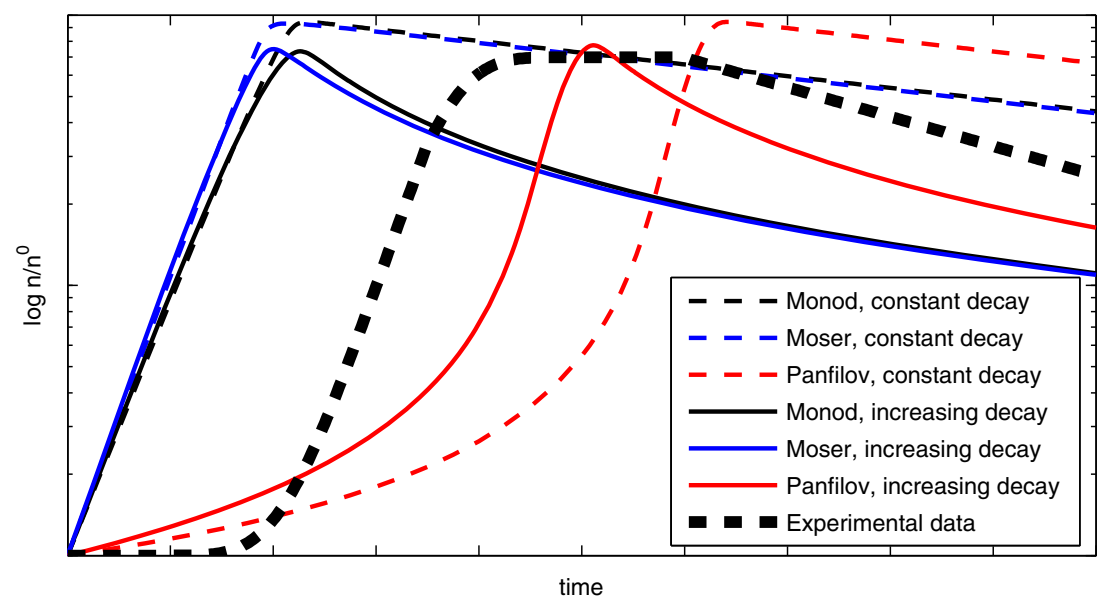


formulation for the competitive inhibition was proposed by [2]. To reduce the complexity, this is not considered in the present model.

\subsection{Coupling of processes}

The coupling of flow, transport, microbial population dynamics, and bio-chemical reactions are required for the derivation of the final model. For the consideration of four microbial processes, represented by the reaction equations (1) to (4), the model includes six mobile and one solid component. The governing system of equations can be found in Table 1 where the subscript $m$ relates the four microbial species: (M) Methanogenic archaea, (A) acetogenic archaea, (S) sulfate-reducing bacteria, and (I) iron-reducing bacteria, the subscripts $s, w$, and $g$ denote the solid, water, and gas phase and the superscript $k$ relates the seven components of the system: $\mathrm{H}_{2}, \mathrm{CO}_{2}, \mathrm{CH}_{4}, \mathrm{H}_{2} \mathrm{O}, \mathrm{CH}_{3} \mathrm{COOH}$, $\mathrm{SO}_{4}^{2-}, \mathrm{Fe}_{2}^{\mathrm{III}} \mathrm{O}_{3}$. The first four can be present in both mobile phases, $\mathrm{SO}_{4}^{2-}$ and $\mathrm{CH}_{3} \mathrm{COOH}$ are only present in the water phase and $\mathrm{Fe}_{2}^{\mathrm{III}} \mathrm{O}_{3}$ only in the solid phase. The reaction products $\mathrm{H}_{2} \mathrm{~S}$ and $\mathrm{Fe}_{3}^{\mathrm{II}} \mathrm{O}_{4}$ are neglected because its amount is assumed to be insignificant for the balance and equilibrium calculations. The stoichiometric coefficients $\gamma_{m}^{k}$ are relating the reaction equations in (mol):

$$
\begin{aligned}
& \gamma_{M}=\left(\begin{array}{c}
-4 \\
-1 \\
1 \\
2 \\
0 \\
0 \\
0
\end{array}\right), \quad \gamma_{A}=\left(\begin{array}{c}
-4 \\
-2 \\
0 \\
2 \\
1 \\
0 \\
0
\end{array}\right), \\
& \gamma_{S}=\left(\begin{array}{c}
-5 \\
0 \\
0 \\
4 \\
0 \\
-1 \\
0
\end{array}\right), \quad \gamma_{I}=\left(\begin{array}{c}
-1 \\
0 \\
0 \\
1 \\
0 \\
0 \\
-3
\end{array}\right)
\end{aligned}
$$

For the microbial growth functions $\psi_{m}^{\text {growth }}$, it is important to consider the availability of the substrate and the corresponding electron acceptor. For this purpose, an extension of the Monod model was initially proposed by [24] which is often called "double Monod model". Related to this, we defined the microbial growth functions as follows:

- for methanogenic archaea:

$\psi_{M}^{\text {growth }}=\psi_{M, \max }^{\text {growth }}\left(\frac{c_{w}^{H_{2}}}{\alpha_{M, 1}+c_{w}^{H_{2}}}\right)\left(\frac{c_{w}^{C O_{2}}}{\alpha_{M, 2}+c_{w}^{C O_{2}}}\right)$

- for acetogenic archaea:

$\psi_{A}^{\text {growth }}=\psi_{A, \max }^{\text {growth }}\left(\frac{c_{w}^{H_{2}}}{\alpha_{A, 1}+c_{w}^{H_{2}}}\right)\left(\frac{c_{w}^{C O_{2}}}{\alpha_{A, 2}+c_{w}^{C O_{2}}}\right)$

- for sulfate-reducing bacteria:

$\psi_{S}^{\text {growth }}=\psi_{S, \max }^{\text {growth }}\left(\frac{c_{w}^{H_{2}}}{\alpha_{S, 1}+c_{w}^{H_{2}}}\right)\left(\frac{c_{w}^{S O_{4}^{2-}}}{\alpha_{S, 2}+c_{w}^{S O_{4}^{2-}}}\right)$

- for iron-reducing bacteria:

$$
\begin{aligned}
\psi_{I}^{\text {growth }} & =\psi_{I, \max }^{\text {growth }}\left(\frac{c_{w}^{H_{2}}}{\alpha_{I, 1}+c_{w}^{H_{2}}}\right) \\
& \cdot\left(\frac{c_{s}^{F e_{2}^{\text {III }} O_{3}}}{\alpha_{I, 2}+c_{s}^{F e_{2}^{I I I} O_{3}}}\right)
\end{aligned}
$$

Each species has its particular empirical parameters which control the rate of growth and consequently the rate of biochemical reaction. An equivalent extension is possible for the other growth models introduced in Section 4.3.

Table 1 Coupled system of equations

- Microbial population dynamics:

$\frac{\partial n_{m}}{\partial t}=S_{w} \psi_{m}^{\text {growth }} \cdot n_{m}-\psi_{m}^{\text {decay }} \cdot n_{m}+\nabla \cdot\left(D_{m} \nabla n_{m}\right), \quad m=M, A, S, I$

- Reactive transport for mobile components:

$\phi \frac{\partial\left(\rho_{g} c_{g}^{k} S_{g}+\rho_{w} c_{w}^{k} S_{w}\right)}{\partial t}+\nabla \cdot\left(-\rho_{g} c_{g}^{k} \frac{K k_{r g}}{\mu_{g}} \cdot\left(\nabla P_{g}-\hat{\rho}_{g} g\right)-\rho_{w} c_{w}^{k} \frac{K k_{r w}}{\mu_{w}} \cdot\left(\nabla P_{w}-\hat{\rho}_{w} g\right)\right)$

$+\nabla \cdot\left(-\left(\sum_{j=1 \neq i}^{n} \frac{c_{g}^{j}}{\rho_{g} D_{\text {diff }, g}^{i j}}\right)^{-1} \nabla c_{g}^{k}-\rho_{g} D_{\text {disp }, g}^{k} \nabla c_{g}^{k}-\rho_{w}\left(D_{\text {diff }, w}^{k}+D_{\text {disp }, w}^{k}\right) \nabla c_{w}^{k}\right)=\phi S_{w} \sum_{m} \gamma_{m}^{k} \frac{\psi_{m}^{\text {growth }}}{Y_{m}} n_{m}$,

$k=\mathrm{H}_{2}, \mathrm{CO}_{2}, \mathrm{CH}_{4}, \mathrm{H}_{2} \mathrm{O}, \mathrm{CH}_{3} \mathrm{COOH}, \mathrm{SO}_{4}^{2-}, \quad m=M, A, S, I$

- Heterogeneous reaction:

$(1-\phi) \frac{\partial\left(\rho_{s} c_{s}^{k}\right)}{\partial t}=\phi S_{w} \gamma_{I}^{k} \frac{\psi_{I}^{\text {growth }}}{Y_{I}} n_{I}, \quad k=F e_{2}^{\mathrm{III}} O_{3}$ 


\subsection{Transformation to dimensionless form}

The following dimensionless parameters were introduced to obtain a dimensionless form:

$$
\begin{aligned}
& \bar{\rho}=\frac{\rho}{\rho^{\star}}, \quad \overline{\hat{\rho}}=\frac{\hat{\rho}}{\hat{\rho}^{\star}}, \quad \bar{\mu}=\frac{\mu}{\mu^{\star}}, \quad \bar{P}=\frac{P}{P^{\star}}, \\
& \bar{D}=\frac{D}{D^{\star}}, \quad \tau=\frac{t}{t^{\star}}, \quad \bar{g}=\frac{g}{g^{\star}}, \quad \bar{x}=\frac{x}{L}, \\
& \bar{n}=\frac{n}{n^{\star}}, \quad \bar{K}=\frac{K}{K^{\star}}, \quad \bar{\psi}^{\text {growth }}=t^{\star} \cdot \psi^{\text {growth }}, \\
& \bar{\psi}^{\text {decay }}=t^{\star} \cdot \psi^{\text {decay }}, \quad \overline{\hat{q}}=\frac{\hat{q}}{\hat{q}^{\star}}
\end{aligned}
$$

where the $\star$ denotes the characteristic value of the parameter. The characteristic time was selected as the time of advective flow:

$t^{\star}=\frac{L^{2} \mu^{\star}}{K^{\star} P^{\star}}$

The resulting dimensionless system of equations is shown in Table 2 where $\beta, \delta$ and $\epsilon$ are dimensionless coefficients:

$\beta=\frac{\mu^{\star} D^{\star}}{K^{\star} P^{\star}}, \quad \delta_{m}^{k}=\frac{n^{\star} \gamma_{m}^{k}}{\rho^{\star} Y_{m}}, \quad \epsilon=\frac{\hat{\rho}^{\star} g^{\star} L}{P^{\star}}$

$\beta$ can be also written as:

$\beta=\frac{t^{\star}}{t^{\star \star}}$

where $t^{\star \star}$ is the characteristic time of diffusion which is defined as:

$t^{\star \star}=\frac{L^{2}}{D^{\star}}$

This means that $\beta$ is the ratio of the time of advection to the time of diffusion which is similar to the reciprocal of the Péclet number. The product $\left(\delta_{m}^{k} \cdot \bar{\psi}_{m}^{\text {growth }}\right)$ relates the time of advection to the time of the bio-chemical reaction and could be defined as the reciprocal of the Damköhler number. $\epsilon$ is the dimensionless gravitational number which is relating the gravitational flow to the advective flow.

\section{Numerical implementation}

The numerical simulation of the mathematical model, presented in the previous sections, was implemented by the use of the open source DuMuX [15] as the base platform. DuMuX is a DUNE [3] module for the simulation of multi-phase multi-component flow and transport in porous media. The DuMuX classes are responsible for computation of fluxes, fluid properties, introducing boundary conditions, closure equations like sum of unity of saturation and concentrations, thermodynamic equilibrium, capillary pressure, intrinsic and relative permeabilities. The transport equations were solved by the use of a model already implemented in DuMuX for multi-phase multi-component flow and transport which is called "MpNc." This model implements $\mathrm{M}$-phase $\mathrm{N}$-component flow of $\mathrm{M}$ compressible and partially miscible fluid phases composed of $\mathrm{N}$ components. The fugacities are the primary variables in this model. The phase appearance/disappearance criteria are implemented through additional complementarity equations on the sum of the molar concentrations in each phase and saturation of each phase. We implemented a new module for microbial population dynamics and bio-chemical reactions and new equations for the components that do not participate in overall thermodynamic equilibrium, such as $\mathrm{SO}_{4}^{2-}$ and $\mathrm{Fe}_{2}^{\mathrm{III}} \mathrm{O}_{3}$. A 2D unstructured grid was used in the format of ALUGrid data [11]. For spatial discretization, the Box-method (vertex-centered finite volume method [20]) was used. This method can be classified as a node-centered finite volume method based on the Galerkin finite element method. Boxmethod unites the advantages of the finite-volume (FV) and finite-element (FE) methods: The advantage of the FE method is that unstructured grids can be used, while the FV method is mass conservative [36]. During the first numerical tests, it was observed that the grid need to be slightly more dense for the coupled bio-chemical model than for a purely hydrodynamic model. High gradients in the number of microorganisms tend to result in instability problems. However, this problem was solved by the selection of an adequate grid density and no further adjustments were

Table 2 Dimensionless coupled system of equations

Microbial population dynamics:

$\frac{\partial \bar{n}_{m}}{\partial \tau}=S_{w} \bar{\psi}_{m}^{\text {growth }} \cdot \bar{n}_{m}-\bar{\psi}_{m}^{\text {decay }} \cdot \bar{n}_{m}+\beta \nabla \cdot\left(\bar{D}_{m} \nabla \bar{n}_{m}\right), \quad m=M, A, S, I$

Reactive transport for mobile components:

$\phi \frac{\partial\left(\bar{\rho}_{g} c_{g}^{k} S_{g}+\bar{\rho}_{w} c_{w}^{k} S_{w}\right)}{\partial \tau}+\nabla \cdot\left(-\bar{\rho}_{g} c_{g}^{k} \frac{\bar{K} k_{r g}}{\bar{\mu}_{g}}\left(\nabla \bar{P}_{g}-\epsilon \overline{\hat{\rho}}_{g} \bar{g}\right)-\bar{\rho}_{w} c_{w}^{k} \frac{\bar{K} k_{r w}}{\bar{\mu}_{w}}\left(\nabla \bar{P}_{w}-\epsilon \overline{\hat{\rho}}_{w} \bar{g}\right)\right)$

$+\beta \nabla \cdot\left(-\left(\sum_{j=1 \neq i}^{n} \frac{c_{g}^{j}}{\bar{\rho}_{g} \bar{D}_{\mathrm{diff}, g}^{i j}}\right)^{-1} \nabla c_{g}^{k}-\bar{\rho}_{g} \bar{D}_{\mathrm{disp}, g}^{k} \nabla c_{g}^{k}-\bar{\rho}_{w}\left(\bar{D}_{\mathrm{diff}, w}^{k}+\bar{D}_{\mathrm{disp}, w}^{k}\right) \nabla c_{w}^{k}\right)=\phi S_{w} \sum_{m} \delta_{m}^{k} \bar{\psi}_{m}^{\text {growth }} \bar{n}_{m}$,

$k=\mathrm{H}_{2}, \mathrm{CO}_{2}, \mathrm{CH}_{4}, \mathrm{H}_{2} \mathrm{O}, \mathrm{CH}_{3} \mathrm{COOH}, \mathrm{SO}_{4}^{2-}, \quad m=\mathrm{M}, \mathrm{A}, \mathrm{S}, \mathrm{I}$

Heterogeneous reaction:

$(1-\phi) \frac{\partial\left(\bar{\rho}_{s} c_{s}^{k}\right)}{\partial \tau}=\phi S_{w} \delta_{I}^{k} \bar{\psi}_{I}^{\text {growth }} \bar{n}_{I}, \quad k=F e_{2}^{\mathrm{III}} O_{3}$ 
required in the discretization method and solver. For the time discretization, a fully implicit Euler scheme is applied which is unconditionally stable and can be applied to complex problems such as the multi-phase flow in porous media. The time-stepping scheme is adaptive which means that the length of the new time step is calculated based on required number of iterations of the previous one. Hence, the time step length automatically adjusts to the time scale of the fastest process. This procedure is very helpful in the context of UHS where it is unknown if advective flow or the biochemical reactions have the smallest time scale. The system was solved fully-coupled to keep it as accurate as possible. The high computational cost and memory are not a limiting factor for the 2D conceptual simulations in Section 6. The system of equations has been linearized using the first expansion of Taylor series neglecting higher-order terms. The resulting linear set of equations was solved with the Newton-Raphson method which is an iterative root-finding algorithm.

\section{Simulation of hydrogen injection into a depleted gas reservoir}

The presented mathematical model was used to study the injection of hydrogen into an depleted gas reservoir. The two-dimensional reservoir geometry represents a vertical slice through an anticline structure (cf. Fig. 3). The initialization was done in hydrodynamic equilibrium wherefore the gas-water contact (GWC) was defined at a certain depth with a dimensionless pressure of 1 . The phase pressures and saturations were calculated by using the pressure gradients and capillary pressure relation. Hydrogen was injected at a constant rate into the top center of the reservoir. The lower and the upper boundaries of the reservoir are assumed to be in no flux condition whereas the left and right boundaries are assumed to be kept as initial condition. The reservoir is homogeneous and isotropic. The characteristic parameters are summarized in Table 3. Related to these parameters, the characteristic time and dimensionless coefficients are defined as:

$t^{\star}=2.5 \cdot 10^{7} s, \quad \beta=1 \cdot 10^{-4}$,

$\delta_{m}^{k}=1 \cdot 10^{-3} \cdot \gamma_{m}^{k}, \quad \epsilon=4.905 \cdot 10^{-2}$

Different simulation cases were run to investigate the hydrodynamic and bio-chemical effects.

\subsection{Hydrodynamic effects}

A purely hydrodynamic study was carried out to investigate the influence of the injection rate. It was assumed

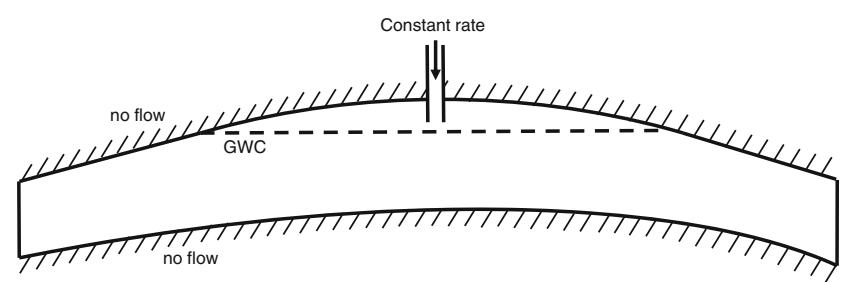

Fig. 3 Sketch of the reservoir geometry with initial and boundary conditions

that microorganisms are absent which means that no biochemical transformations took place. The initial gas composition in this case was $100 \% \mathrm{H}_{2}$ and the injection of hydrogen was studied at ten different injection rates, reaching from very low to very high. In addition, a comparison to a natural gas storage was done. Therefore, the injection of methane was studied at the same rate whereby also the initial gas composition was $100 \% \mathrm{CH}_{4}$. In Fig. $4 \mathrm{a}$, the initial gas saturation is plotted. Due to the symmetry of the reservoir, it suffices to show the left half. The interpretation of the saturation profiles after a certain period of injection indicates that three different displacement regimes could arise.

Figure $4 \mathrm{~b}$ shows the gas saturation for a very slow hydrogen injection rate $\left(\overline{\hat{q}}=1 \cdot 10^{-4}\right)$. The characteristic injection rate $\hat{q}^{\star}$ is defined as $1000 \frac{\mathrm{mol}}{\mathrm{s}}$. In this case, the gravitational forces are dominating. The GWC lowers evenly and the gas saturation in the displaced region is very high. The injection of methane at the same rate has shown exactly the same saturation profile.

In Fig. 4c, the saturation is shown for a medium injection rate $\left(\overline{\hat{q}}=2 \cdot 10^{-3}\right)$. For this injection rate, the behavior was influenced by a combined action of gravitational and viscous forces. The GWC lowers only slightly in the center of the reservoir while the gas is spreading laterally below the cap rock.

Table 3 List of characteristic parameters

\begin{tabular}{llll}
\hline Parameter & Symbol & Value & Unit \\
\hline Molar density & $\rho^{\star}$ & 5000 & $\mathrm{~mol} / \mathrm{m}^{3}$ \\
Density & $\hat{\rho}^{\star}$ & 10 & $\mathrm{~kg} / \mathrm{m}^{3}$ \\
Dynamic viscosity & $\mu^{\star}$ & $1 \cdot 10^{-5}$ & $\mathrm{~Pa} \cdot \mathrm{s}$ \\
Pressure & $P^{\star}$ & $6 \cdot 10^{6}$ & $\mathrm{~Pa}$ \\
Diffusion coefficient & $D^{\star}$ & $1 \cdot 10^{-6}$ & $\mathrm{~m} / \mathrm{s}$ \\
Length & $L$ & 500 & $\mathrm{~m}$ \\
Gravity acceleration & $g^{\star}$ & 9.81 & $\mathrm{~m} / \mathrm{s}^{2}$ \\
Porosity & $\phi$ & 0.2 & - \\
Permeability & $K^{\star}$ & 100 & $\mathrm{mD}$ \\
Pore size distribution index & $\lambda$ & 2 & - \\
Entry capillary pressure & $P_{e}$ & $1 \cdot 10^{5}$ & $\mathrm{~Pa}$ \\
\hline
\end{tabular}




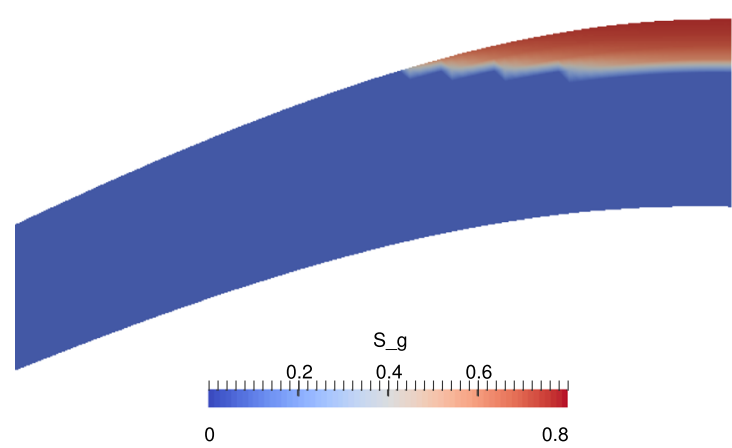

(a) $S_{g}$ at $\tau=0$

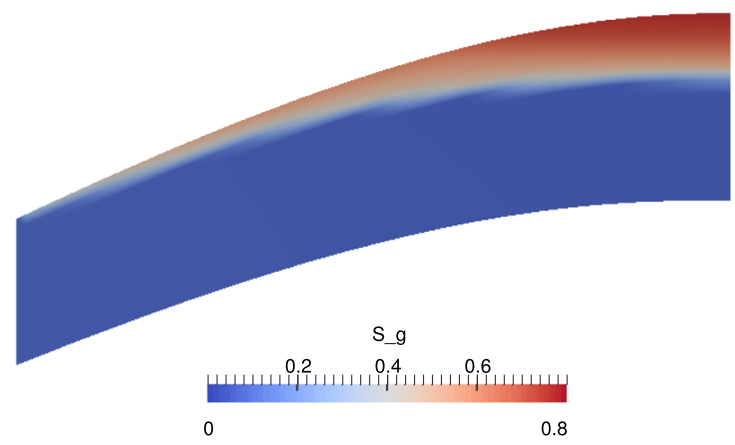

(c) Hydrogen injection, $S_{g}$ at $\tau=1.04$ for $\bar{q}=2 \cdot 10^{-3}$

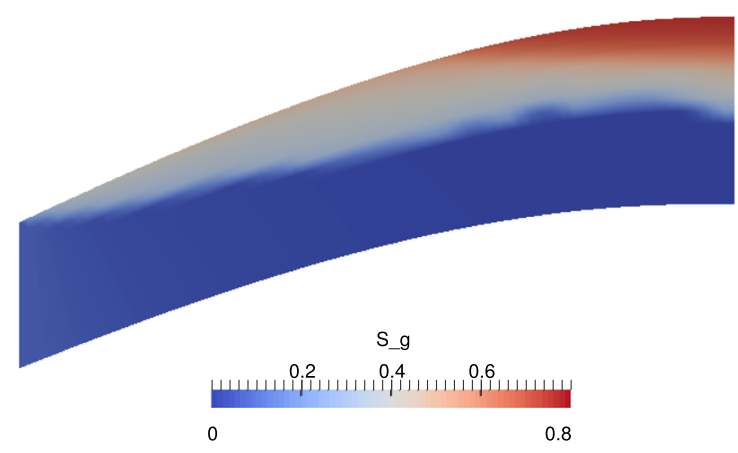

(e) Hydrogen injection, $S_{g}$ at $\tau=0.128$ for $\bar{q}=0.1$

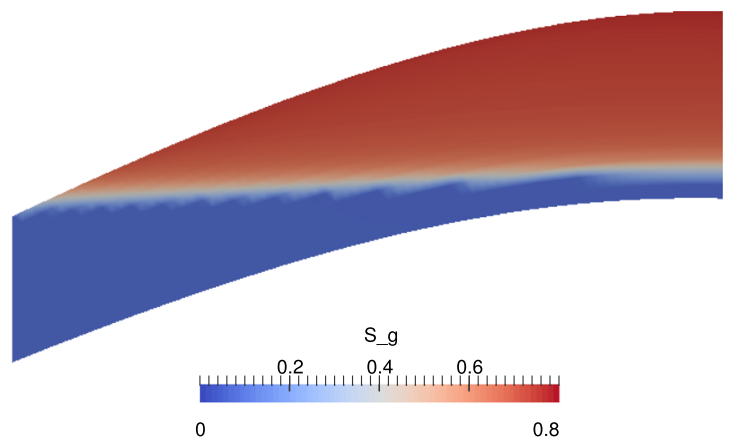

(b) Hydrogen injection, $S_{g}$ at $\tau=108$ for $\bar{q}=1 \cdot 10^{-4}$

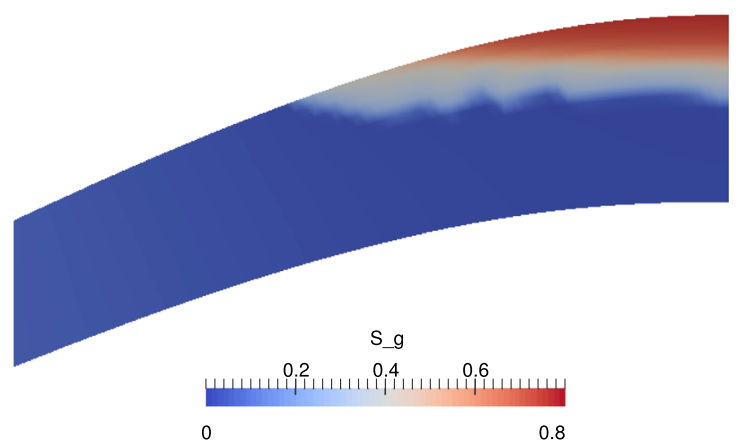

(d) Hydrogen injection, $S_{g}$ at $\tau=0.05$ for $\bar{q}=0.1$

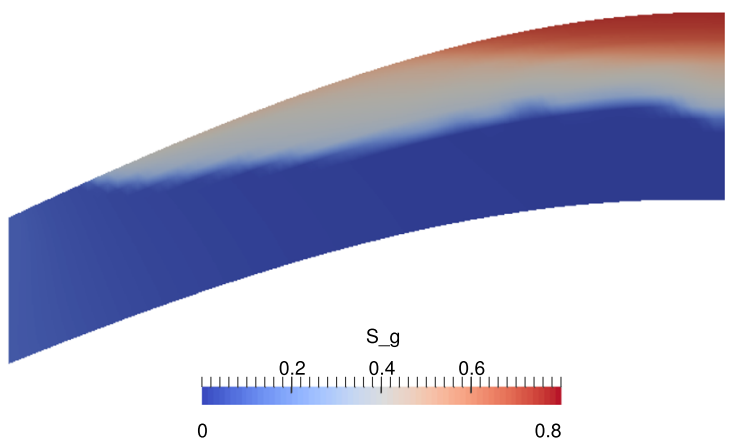

(f) Methane injection, $S_{g}$ at $\tau=0.128$ for $\bar{q}=0.1$

Fig. 4 Purely hydrodynamic simulation of hydrogen injection into the top center of a conceptional two-dimensional anticline structure

For high injection rates $(\overline{\hat{q}}=0.1)$, the viscous forces are dominating. In Fig. $4 \mathrm{~d}$, the gas saturation is shown after a short time of hydrogen injection. At the beginning, the GWC lowers more or less evenly. However, the gas saturation behind the displacement front is relatively low (grey region). After a certain time, the lowering in the center almost stops and lateral gas fingers start to propagate towards the flanks of the structure (cf. Fig. 4e).

The comparison to the injection of methane (cf. Fig. 4f) shows that hydrogen spreads laterally faster. The cause is the more unfavorable mobility ratio due to low dynamic viscosity of hydrogen.

\subsection{Bio-chemical effects}

A different study was performed for the investigation of biochemical effects in UHS. In this study, the microbial growth was described by the "double Monod model" and the decay by an increasing decay rate. The depleted reservoir contains a residual amount of a mixed gas $\left(80 \% \mathrm{CH}_{4}\right.$ and $20 \%$ $\mathrm{CO}_{2}$ ). The initialization and boundary conditions are identical to the previous description. The injection rate was $\overline{\hat{q}}=$ $1 \cdot 10^{-3}$. The dimensionless parameters for the microbial population dynamics used in this study are summarized in Table 4; however, in the current state, these parameters are 
Table 4 List of dimensionless parameters for microbial population dynamics

\begin{tabular}{lll}
\hline Dimensionless parameter & Symbol & Value \\
\hline Maximum specific growth rate & $\bar{\psi}_{M, \text { max }}^{\text {growth }}$ & 50 \\
Decay coefficient & $\bar{d}$ & 1 \\
$\mathrm{H}_{2}$ half-velocity constant & $\alpha_{M, 1}$ & $1 \cdot 10^{-4}$ \\
$\mathrm{CO}_{2}$ half-velocity constant & $\alpha_{M, 2}$ & $1 \cdot 10^{-3}$ \\
Microbial diffusion coefficient & $\bar{D}_{m}$ & 0.1 \\
\hline
\end{tabular}

estimated with a very high uncertainty. An exact determination from laboratory experiments is planned in future

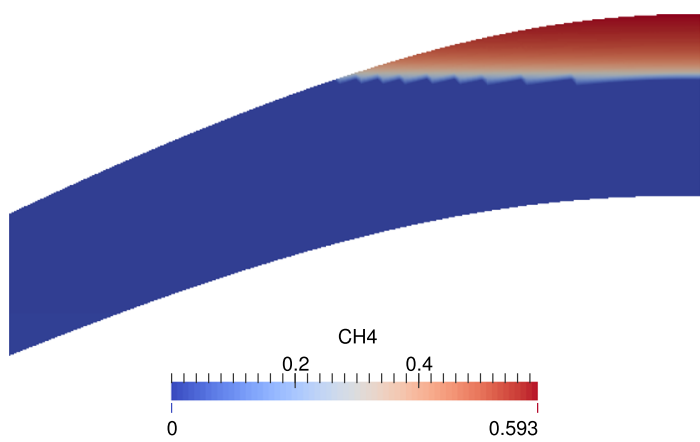

(a) Initial total $\mathrm{CH}_{4}$ concentration

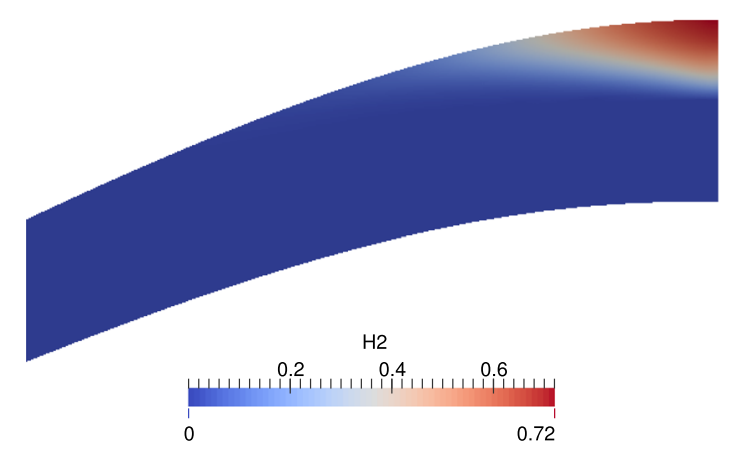

(c) Final total $\mathrm{H}_{2}$ concentration for the case without biochemical reactions

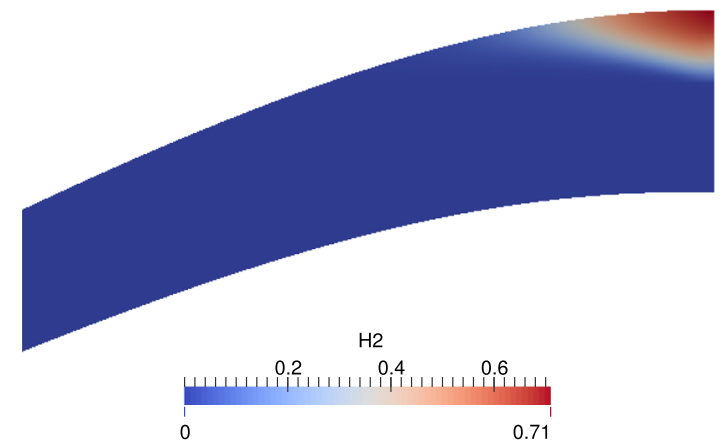
(e) Final total $H_{2}$ concentration for the case with methanogen-
esis research projects. Two simulation cases were executed and compared: (1) without any bio-chemical reaction, and (2) with methanogenesis. In Fig. 5a, the initial methane concentration is shown.

Figure $5 \mathrm{~b}-\mathrm{e}$ are showing the results after an injection period of $\tau=1.3$. The plots of concentrations respectively for $\mathrm{CH}_{4}$ and $\mathrm{H}_{2}$ are compared for both cases. In both cases, the hydrogen propagates around the injection well whereby it tends to spread below the cap rock. However, some differences are recognizable. The concentration front is much more diffusive in the case without methanogenesis. Additionally, by comparing Fig. 5c and e, it can be identified

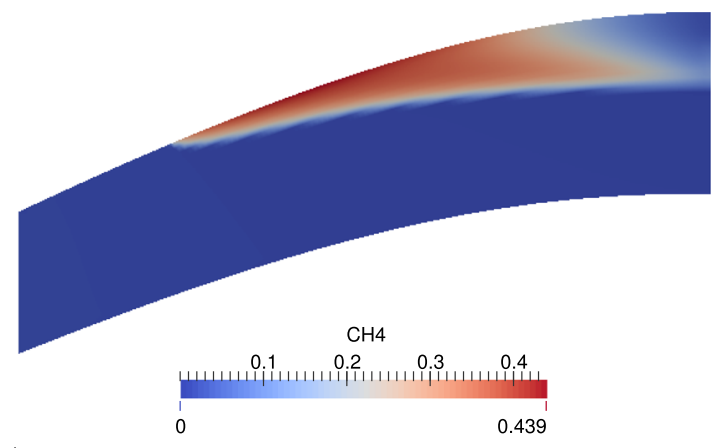

(b) Final total $\mathrm{CH}_{4}$ concentration for the case without biochemical reactions

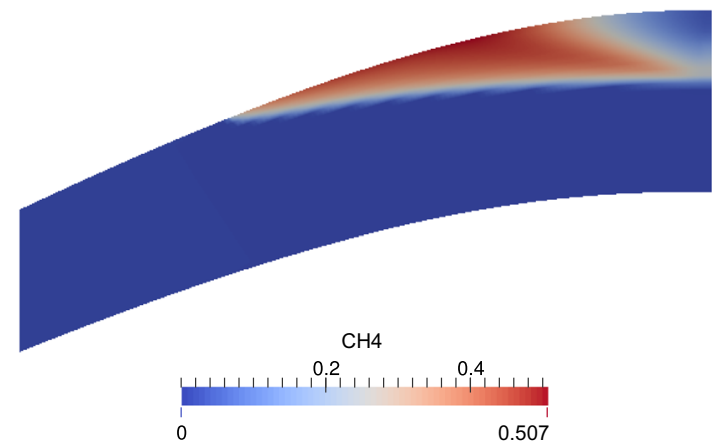

(d) Final total $\mathrm{CH}_{4}$ concentration for the case with methanogenesis

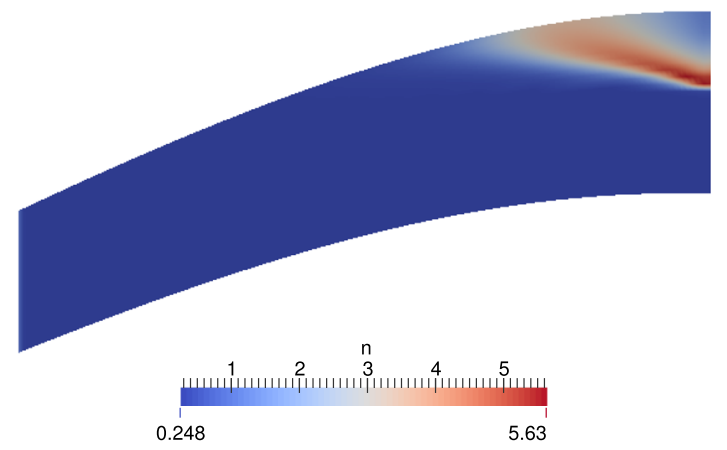

(f) Dimensionless number of microorganisms after $\tau=1.3$ for Monod model

Fig. 5 Coupled bio-chemical and hydrodynamic simulation of hydrogen injection into the top center of a conceptional two-dimensional anticline structure 


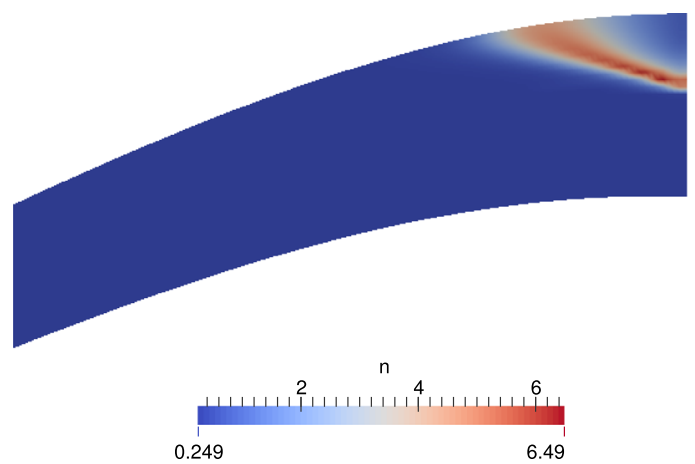

(a) Dimensionless number of microorganisms after $\tau=1.3$ for Moser model

Fig. 6 Comparison of different models for the microbial growth

that the spreading in the case with methanogenesis is less. The explanation for this behavior is the microbial activity at the hydrogen concentration front. At this diffusive front, the methanogenic archaea are exposed to both substrates $\left(\mathrm{H}_{2}\right.$ and $\left.\mathrm{CO}_{2}\right)$. Hence, an intense growth of microorganisms takes place (cf. Fig. 5f) and the injected $\mathrm{H}_{2}$ is partially transformed into $\mathrm{CH}_{4}$ and $\mathrm{H}_{2} \mathrm{O}$. Consequently, the spreading of the injected gas is slowed down.

\subsection{Comparison of different models for microbial growth}

In Section 4.3, it was already shown that the selection of the microbial growth model has an important role in the simulation of batch culture experiments. In this part of the simulation study, the different growth models are used and compared in the coupled hydrodynamic and bio-chemical simulation. The case study corresponds to Section 6.2 and only the growth model was changed. In Fig. $6 a, b$, the dimensionless number of microorganisms is shown after the injection period for the Moser and the Panfilov model. Compared to Fig. 5f, there are significant differences in the predicted growth of microorganisms.

\section{Conclusions}

- Hydrogen is a universal electron donor for the metabolism of different microbial species which are present in subsurface structures. Hence, the injection of hydrogen stimulates their activity and problems could arise. Four different hydrogenotrophic species could be important for UHS: Methanogenic archaea, acetogenic archaea, sulfate-reducing bacteria, and iron-reducing bacteria. The coexistence of several species can thereby result in a competition for hydrogen.

- The local rate of the bio-chemical reactions depends on the number of the particular microorganism. Hence,

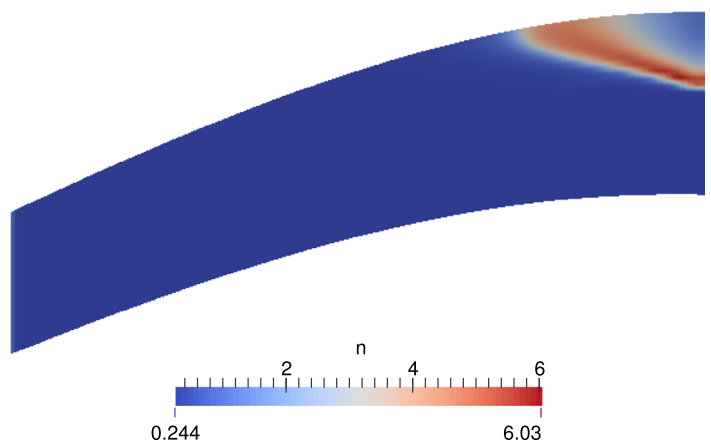

(b) Dimensionless number of microorganisms after $\tau=1.3$ for Panfilov model an important problem for the modeling of UHS is the description of microbial growth and decay functions.

- A mathematical model was presented which describes the hydrodynamic behavior of UHS coupled with biochemical reactions and microbial population dynamics. It considers the metabolism of four hydrogenotrophic microbial species and includes the flow and transport of six components in two mobile phases and one rock component.

- A comparison of three substrate-limited growth models and two decay models is presented. None of the models shows the lag and stationary phases which have been observed in batch culture experiments. Only the model of [29] shows the acceleration phase.

- The purely hydrodynamic simulation study has shown the importance of the injection rate. At high injection rate the displacement of water becomes unstable and lateral fingers spread below the cap rock. It was shown that lateral fingers in UHS spread faster than in storages of natural gas. At low injection rates, the displacement is stable and the GWC lowers uniformly.

- The simulation study with coupled bio-chemical processes has shown some significant differences for the two cases with and without methanogenesis. A growth of microorganisms arises at the front where the injected hydrogen gas displaces the initial gas. The increased number of microorganisms results in a partial transformation of the injected $\mathrm{H}_{2}$ into $\mathrm{CH}_{4}$ and $\mathrm{H}_{2} \mathrm{O}$. Additionally, the selection of the microbial growth function has an important influence.

Open Access This article is distributed under the terms of the Creative Commons Attribution 4.0 International License (http:// creativecommons.org/licenses/by/4.0/), which permits unrestricted use, distribution, and reproduction in any medium, provided you give appropriate credit to the original author(s) and the source, provide a link to the Creative Commons license, and indicate if changes were made. 


\section{References}

1. Allison, D.G.: Community Structure and Co-operation in Biofilms, vol. 59. Cambridge University Press (2000)

2. Bailey, J.E., Ollis, D.F.: Biochemical engineering fundamentals. Chemical Engineering Education (1976)

3. Bastian, P., Blatt, M., Dedner, A., Engwer, C., Klöfkorn, R., Ohlberger, M., Sander, O.: A generic grid interface for parallel and adaptive scientific computing. part I: abstract framework. Computing 82(2-3), 103-119 (2008)

4. Brooks, RH., Corey, T.: Hydraulic properties of porous media (1964)

5. Bulatov, G.: Underground storage of hydrogen. $\mathrm{PhD}$ thesis, Moscow Gubkin Oil and Gas University (in Russian) (1979)

6. Carden, P., Paterson, L.: Physical, chemical and energy aspects of underground hydrogen storage. Int. J. Hydrog. Energy 4(6), 559569 (1979)

7. Chen, Z.: Reservoir simulation: mathematical techniques in oil recovery, vol. 77. SIAM (2007)

8. Cord-Ruwisch, R., Seitz, H.J., Conrad, R.: The capacity of hydrogenotrophic anaerobic bacteria to compete for traces of hydrogen depends on the redox potential of the terminal electron acceptor. Arch. Microbiol. 149(4), 350-357 (1988)

9. Crotogino, F., Donadei, S., Bünger, U., Landinger, H.: Largescale hydrogen underground storage for securing future energy supplies. In: 18th World Hydrogen Energy Conference, pp. 16-21 (2010)

10. Scheidegger, A.E.: The Physics of Flow Through Porous Media, Soil Science, vol. 86, p. 355. LWW (1958)

11. Dedner, A., Klöfkorn, R., Nolte, M.: The DUNE-ALUGrid module. arXiv:14076954 (2014)

12. Dufrenne, J., Delfgou, E., Ritmeester, W., Notermans, S.: The effect of previous growth conditions on the lag phase time of some foodborne pathogenic micro-organisms. Int. J. Food Microbiol. 34(1), 89-94 (1997)

13. DVGW: Arbeitsblatt G262: Nutzung von Gasen aus regenerativen Quellen in der öffentlichen Gasversorgung (2011)

14. Ebigbo, A., Golfier, F., Quintard, M.: A coupled, pore-scale model for methanogenic microbial activity in underground hydrogen storage. Adv. Water Resour. 61, 74-85 (2013)

15. Flemisch, B., Darcis, M., Erbertseder, K., Faigle, B., Lauser, A., Mosthaf, K., Müthing, S., Nuske, P., Tatomir, A., Wolff, M., et al.: DuMu${ }^{x}$ : DUNE for multi-phase, component, scale, physics, ... flow and transport in porous media. Adv. Water Resour. 34(9), 1102-1112 (2011)

16. Foh, S.E.: Underground Hydrogen Storage: Final Report. Brookhaven National Laboratory, Department of Energy and Environment (1979)

17. Ganser, C., Eng, B.: New energy storage concept for renewable energies in the form of potential energy storage, p. 70. Techniken zur Energiewende (2013)

18. Ginn, T.R., Wood, B.D., Nelson, K.E., Scheibe, T.D., Murphy, E.M., Clement, T.P.: Processes in microbial transport in the natural subsurface. Adv. Water Resour. 25(8), 1017-1042 (2002)

19. Golfier, F., Wood, B.D., Orgogozo, L., Quintard, M., Buès, M.: Biofilms in porous media: development of macroscopic transport equations via volume averaging with closure for local mass equilibrium conditions. Adv. Water Resour. 32(3), 463-485 (2009)

20. Helmig, R. et al.: Multiphase flow and transport processes in the subsurface: a contribution to the modeling of hydrosystems. Springer (1997)

21. Kepplinger, J., Crotogino, F., Donadei, S., Wohlers, M.: Present trends in compressed air energy and hydrogen storage in germany. In: Solution Mining Research Institute SMRI Fall 2011 Conference, York, United Kingdom (2011)

22. Kleinitz, W., Boehling, E.: Underground gas storage in porous media-operating experience with bacteria on gas quality (spe94248) (2005)

23. Lovley, D.R., Phillips, E.J.P.: Competitive mechanisms for inhibition of sulfate reduction and methane production in the zone of ferric iron reduction in sediments. Appl. Environ. Microbiol. 53(11), 2636-2641 (1987)

24. Megee III, R.D., Drake, J.F., Fredrickson, A., Tsuchiya, H.G.: Studies in intermicrobial symbiosis. Saccharomyces cerevisiae and lactobacillus casei. Can. J. Microbiol. 18(11), 1733-1742 (1972)

25. Monod, J.: The growth of bacterial cultures. Annu. Rev. Microbiol. 3(1), 371-394 (1949)

26. Moser, A.: Bioprocess technology: kinetics and reactors. Springer (1988)

27. Müller-Syring, G., Henel, M., Krause, H., Rasmusson, H., Mlaker, H., Köppel, W., Höcher, T., Sterner, M., Trost, T.: Power-to-gas: Entwicklung von Anlagenkonzepten im Rahmen der DVGWInnovationsoffensive, pp. 770-777. Artikel aus gwf-Gas/Erdgas November (2011)

28. Murphy, E.M., Ginn, T.R.: Modeling microbial processes in porous media. Hydrogeol. J. 8(1), 142-158 (2000)

29. Panfilov, M.: Underground storage of hydrogen: in situ selforganisation and methane generation. Transp. Porous Media 85(3), 841-865 (2010)

30. Paterson, L.: The implications of fingering in underground hydrogen storage. Int. J. Hydrog. Energy 8(1), 53-59 (1983)

31. Poling, B.E., Prausnitz, J.M., John Paul, O., Reid, R.C.: The properties of gases and liquids, vol. 5. McGraw-Hill, New York (2001)

32. Roads2HyCom: Large Hydrogen Underground Storage (2008). www.roads2hy.com

33. Šmigáň, P., Greksak, M., Kozánková, J., Buzek, F., Onderka, V., Wolf, I.: Methanogenic bacteria as a key factor involved in changes of town gas stored in an underground reservoir. FEMS Microbiol. Lett. 73(3), 221-224 (1990)

34. Toleukhanov, A., Panfilov, M., Panfilova, I., Kaltayev, A.: Bio-reactive two-phase transport and population dynamics in underground storage of hydrogen: Natural self-organisation. In: ECMOR XIII-13th European Conference on the Mathematics of Oil Recovery (2012)

35. Truche, L., Jodin-Caumon, M.C., Lerouge, C., Berger, G., Mosser-Ruck, R., Giffaut, E., Michau, N.: Sulphide mineral reactions in clay-rich rock induced by high hydrogen pressure. Application to disturbed or natural settings up to $250^{\circ} \mathrm{C}$ and $30 \mathrm{bar}$. Chem. Geol. 351, 217-228 (2013)

36. University of Stuttgart: DuMux Handbook (2013) 